\title{
Extraordinary lifespans in ants: a test of evolutionary theories of ageing
}

\section{Laurent Keller ${ }^{\star} \dagger$ \& Michel Genoud* \\ * Institute of Zoology and Animal Ecology, University of Lausanne, Bâtiment de Biologie, 1015 Lausanne, Switzerland \\ $\dagger$ Institute of Zoology, University of Bern, Ethologische Station Hasli, 3032 Hinterkappelen, Switzerland}

Senescence presents not only a medical problem, but also an evolutionary paradox because it should be opposed by natural selection. Evolutionary hypotheses propose that ageing evolves as the necessary cost of processes increasing early reproductive success $^{1,2}$, or because of weaker selection against late-acting mutations ${ }^{3}$. A prediction of these hypotheses is that the rate of ageing should increase and the average lifespan decrease as the rate of extrinsic mortality increases ${ }^{1-7}$. Alternatively, non-adaptive, purely mechanistic hypotheses invoke damage to DNA, cells, tissues and organs as being the unique cause of senescence and ineluctable death of organisms ${ }^{8}$. Here we show that the evolution of eusociality is associated with a 100-fold increase in insect lifespan. Such an increase is predicted by evolutionary theories because termite, bee and ant queens live in colonies that are sheltered and heavily defended against predators. Moreover, a comparison of ants with contrasting life histories also reveals an association between lifespan and extrinsic rate of mortality. These results provide strong support for evolutionary theories of ageing, as purely mechanistic hypotheses of senescence do not propose any association between the rate of extrinsic mortality and lifespans.

The physiological deterioration of organisms as they age presents an evolutionary paradox: if organisms can function well in youth, why can they not continue to do so in old age $e^{5}$ ? Evolutionary and non-adaptive, purely mechanistic hypotheses have been developed separately to account for the apparently universal occurrence of ageing in vertebrates and arthropods ${ }^{2}$ and, with the exception of some experimental studies conducted with several Drosophila species, few attempts have been made to distinguish them ${ }^{2,9}$. Evolutionary hypotheses predict that ageing rate should generally increase and average lifespan should decrease as the rate of age- and condition-independent (extrinsic) mortality increases and that it should be higher in those organisms whose fecundity does not increase markedly after maturity compared to those showing such an increase $e^{1,3-6,10}$. In contrast, and all else being equal, purely physiological theories of senescence predict no relationship between extrinsic mortality, age-dependent fecundity, and rate of senescence. Instead, they frequently predict an association between duration of life and metabolic rate, whereby organisms with lower metabolic rates should have greater lifespans ${ }^{8,11}$. It is important to note that evolutionary hypotheses do not deny that proximate mechanisms such as DNA damage are responsible for ageing: they only predict that natural selection will act on these proximate mechanisms. For example, a lower rate of extrinsic mortality should decrease the frequency of late-acting genes that reduce the efficiency of DNA repair and cause greater DNA damage.

Owing to their peculiar life history, highly eusocial insects with specialized worker and queen morphological castes (ants, termites and the honeybee) provide a unique opportunity to disentangle evolutionary and mechanistic hypotheses for ageing. Mortality of adult queens due to external factors such as predation is low because the queen is sheltered in a heavily defended nest. Hence, the queen is usually immune to predators beyond the nest-founding stage ${ }^{12}$, except in species with unstable nests (see below). Moreover, because colonies typically start producing reproductives (males and new queens) only when the colony has reached a given size, queens experience a dramatic age-dependent increase in reproductive success when they initiate a new colony independently (without the help of workers). In such independent founding species, queens generally produce the first fertile offspring only when they are 3 to 4 years old ${ }^{12}$, thus delaying the onset of ageing. Furthermore, the number of reproductives produced increases with colony size, which in turn correlates with the queen age ${ }^{12}$. Evolutionary theories thus predict that queens of highly eusocial species such as ants, termites and honeybees should exhibit low rates of ageing and long lifespans. A further advantage of studying ageing in eusocial insects is that contrasting life histories often occur among species of similar body mass. Body mass has been shown to be correlated with lifespan and it is therefore a potentially confounding variable in comparative analyses of longevity ${ }^{6,13}$.

As predicted by evolutionary theories, the lifespan of adult eusocial insect queens is much longer than that of adult solitary insects: the mean average longevities of ant, termite and honeybee queens is $10.0 \pm 6.6(n=51), 11.5 \pm 5.4(n=9)$, and 5.6 years, respectively (overall mean for eusocial insects $10.1 \pm 6.4$ years; $n=61$ ), whereas that of 87 solitary insect species from eight orders is only $0.1 \pm 0.2$ years (Fig. 1 ). To remove the problem of phylogenetic non-independence between species ${ }^{14,15}$, we compared the lifespan of eusocial insects with that of other insects by using an independent contrasts approach ${ }^{14,16}$. This analysis reveals a significant correlation between longevity and eusociality $(P=0.02)$ among the 148 species for which data were available (Fig. 2). Purely mechanistic hypotheses cannot satisfactorily explain this difference. Ant, termite and honeybee queens are unlikely to have lower metabolic rates than non-eusocial insects which could account for such a difference: queens produce hundreds or even thousands of eggs every day which may represent more than their own body weight. This enormous reproductive effort must be associated with a high metabolic rate in the queen ${ }^{12}$.

Additional support for the evolutionary hypotheses comes from the comparison among ant species with alternative reproductive strategies. Ant colonies may contain one (monogyny) or several queens (polygyny) ${ }^{17}$. Interspecific variation in queen number is generally associated with differences in nest-founding strategies. Queens of monogynous species typically initiate new colonies independently (that is, without the help of workers), whereas queens of polygynous species generally return or remain in an established colony after mating ${ }^{18-21}$. The high risk of a colony losing

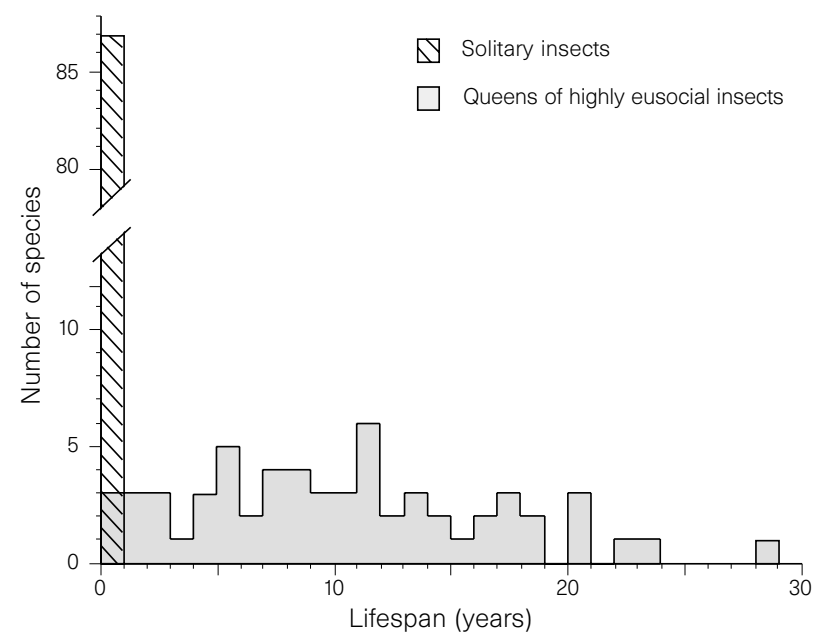

Figure 1 Mean lifespans of 87 solitary insect species from eight orders and 61 species of eusocial insects. We found data on queen lifespan in 51 ant species (Fig. 3), nine termite species and the honeybee. 

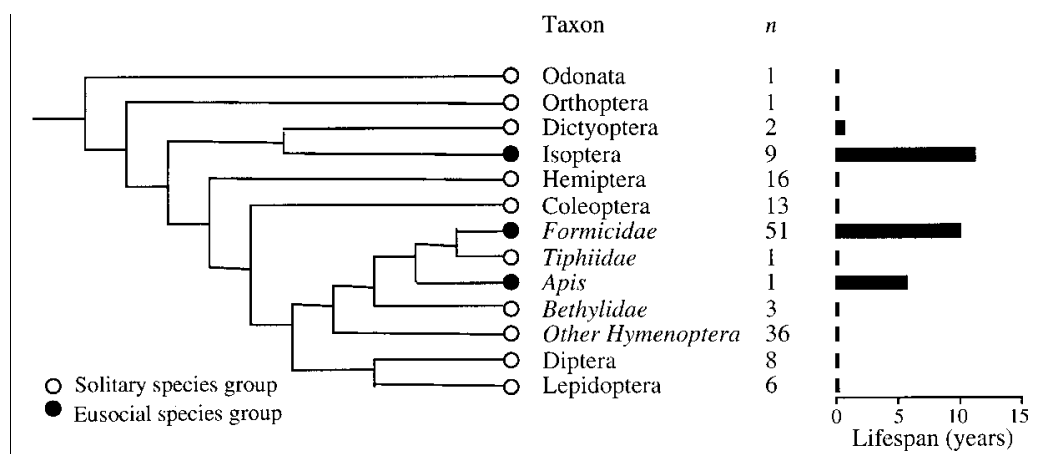

Figure 2 Average lifespans of adults from eight solitary orders of insects and queens from three eusocial insect taxa. Details on the order Hymenoptera is given for several families because eusociality evolved independently in the genus Apis and in Formicidae (ants). The complete phylogeny used for the comparative analysis of independent contrasts is available from L.K.

a queen is one of the major selective forces favouring polygyny over monogyny ${ }^{22,23}$. For instance, polygyny is common in species that do not produce complex sheltered nests and frequently change nest location in response to environmental variation ${ }^{12,20}$. Thus, in polygynous species the risk of extrinsic rate of mortality in mature colonies should be higher than in monogynous species. Moreover, queens returning to established nests after mating may produce sexuals sooner than queens that found independently ${ }^{18}$, hence their reproductive success is less age-dependent (see above).

Evolutionary hypotheses thus predict that a higher lifespan should be associated with monogyny and independent colony founding than with polygyny and dependent colony founding. In accord with this prediction, the average lifespan of queens is much greater in monogynous ( $12.3 \pm 5.5$ years; $n=37)$ than polygynous $(1.6 \pm 1.8$ years; $n=6)$ species. The lifespan of queens in species with both monogynous and polygynous colonies is intermediate (5.7 \pm 6.6 years; $n=8)$. Queen lifespan is also much greater in independent $(12.2 \pm 5.5$ years; $n=38)$ than dependent $(1.7 \pm$ 1.6 years; $n=7$ ) founding species, those species with mixed strategies exhibiting again intermediate values $(5.4 \pm 7.2$ years; $n=6)$. Evolutionary changes in lifespan appear to have accompanied changes in queen number and mode of colony founding on several independent occasions (Fig. 3). An analysis correcting for the effect of phylogeny reveals that both queen number $(P=0.001)$ and mode of colony founding $(P=0.0004)$ are significantly correlated with lifespan. Because variation in queen number is tightly correlated with the mode of colony founding (Fig. 2), it is impossible to disentangle the relative effects of these two factors. However, this is not a problem because the evolutionary hypotheses predict that both monogyny and independent colony founding should select for increased lifespan, the pattern that our analysis revealed. Again, purely mechanistic hypotheses fail to explain this pattern. Although polygynous species change nest location much more frequently than monogynous species, such changes occur only a few times every year, which should not significantly affect the metabolic rate of queens. On the other hand, monogynous queens are usually much more fecund than their polygynous counterparts ${ }^{17-20}$, thus possibly having an even higher metabolic rate.

Circumstantial evidence that longer maximum lifespan occurs in organisms that have lower mortality rates in the wild comes from the comparison of distantly related taxa. Birds and bats, which evade terrestrial predators by flight, generally have greater potential longevities than similarly sized terrestrial vertebrates (refs. 1, 2, but see ref. 24). Similarly, animals with thick shells, such as turtles and bivalves, tend to live longer than animals without armour ${ }^{1,2}$. High maximum lifespans are also found in organisms whose fertility increases with age, such as fish and trees, as predicted by evolutionary theor $y^{2}$. However, as already pointed out ${ }^{2,5,6}$, these wide comparisons have shortcomings and should be considered as consistent with evolutionary theories of ageing rather than as rigorous tests. There are also experimental data supporting evolutionary theories of ageing, although their interpretation has been disputed. In experiments where lineages of Drosophila were selected for late reproduction, longevity was increased ${ }^{2,25}$. This response, which is predicted by evolutionary theories of ageing, is not easily explained by mechanistic theories.

In conclusion, our results offer a comparative analysis of lifespan data for eusocial insects which provides strong support for evolutionary but not purely mechanistic theories of ageing. The decrease in mortality rate and age-dependent increase in fecundity that has accompanied the evolution of eusocial life in ants and termites has

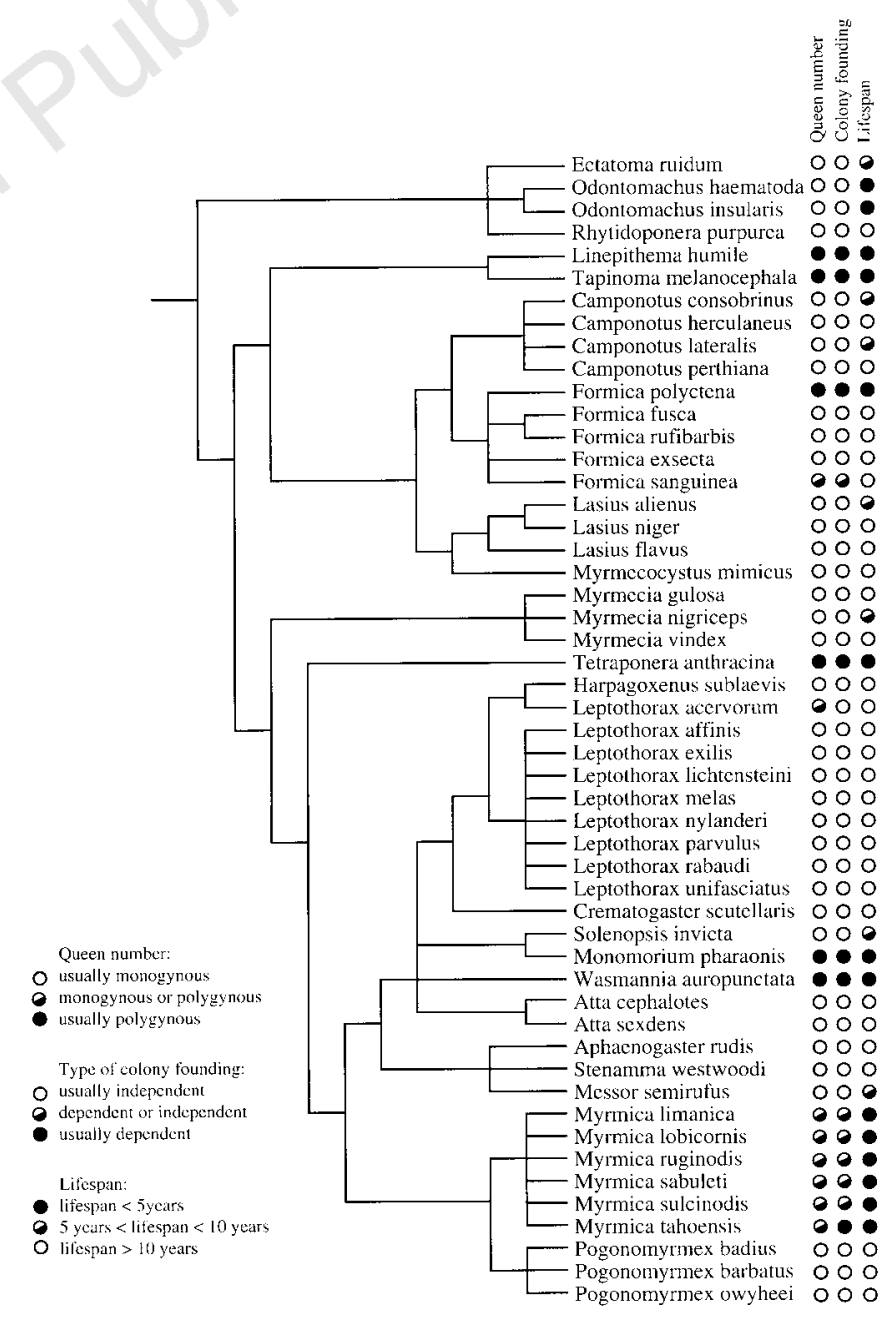

Figure 3 Average lifespans of ant queens in relation to their social organization. Species were classified as monogynous when nests always contain a single queen and as polygynous when nests contain several queens. Species in which both monogynous and polygynous nests occur were classified in an intermediate (mixed) category. Similarly, we differentiated three types of species depending on whether colony founding is always independent, always dependent or whether a mixture of both strategies occurs. References on the mode of colony founding and queen number per colony are available from L.K. 
resulted in an over-100-fold increase in average maximum lifespan, with astonishing values of over 28 years for some ant species. Interestingly, the extrinsic mortality of the only known eusocial mammal, the naked mole-rat, is also low as a result of its subterranean life, and accordingly, the queens of this species live longer than any other rodent of comparable size ${ }^{26}$. Finally, the comparison between ants with alternative life histories supports the assertion that differences in mortality rates and age-dependent fecundities, rather than any other factor associated with the evolution of social life, is responsible for these extreme lifespans.

\section{Methods}

Comparative analyses. We used the CAIC (comparative analysis of independent contrasts) program, version 2.0.0 (ref. 16), which is based on the independent contrasts approach first proposed by Felsenstein ${ }^{14}$. Because our present knowledge of insect diversity is far from being exhaustive, only species used in our analysis were used to construct the phylogeny. Branch lengths were considered equal, which corresponds to a punctuational view of evolutionary change. Under this assumption, the CAIC performs acceptably well even when the phylogeny is poorly resolved ${ }^{27}$. Standardization of the contrasts was checked according to the recommendations in ref. 28. All variables (independent and dependent) were considered continuous. For comparison among insects with different levels of eusociality, 'eusociality' took the value 1 for solitary insects and 2 for eusocial species. For the comparison among ants with contrasting life histories, values ranging between 1 and 3 were assigned to both queen number (monogynous, mixed or polygynous) and mode of colony founding (independent, mixed or dependent). Lifespan data. In three ant and two termite species, only maximum lifespans of queens were given. For these species, we estimated mean lifespan by dividing maximum lifespan by 1.45 , the ratio between maximum and average lifespan observed in Aphaenogaster rudis and Myrmecia vindex (the two species with the largest data set). In some cases the average lifespan is a minimum value as the age of the queen was unknown when the observations began and as queens were still alive when the data were published. Queens were apparently still reproducing just before their death. Data on the lifespan of non-eusocial insect species come from Ridley ${ }^{29,30}$, the only large data set on insect lifespan. These data come from laboratory studies with almost natural rearing conditions. Most of the data on queen lifespan come from laboratory studies, but a minority of the data are from field studies (in 3 genera). Because extrinsic mortality may have occurred, these studies may yield an underestimate of average lifespan. This makes the comparison between eusocial and noneusocial species conservative. For comparison between ants with contrasting life histories, we also conducted the comparative analysis of independent contrasts without considering species in which lifespan was estimated in the field. The exclusion of these species did not affect the significance of the tests performed. Exact lifespans of each species and references are available from L.K.

Received 23 May; accepted 25 July 1997.

1. Williams, G. C. Pleitropy, natural selection, and the evolution of senescence. Evolution 11, 398-44 (1957).

2. Rose, M. Evolutionary Biology of Aging (Oxford Univ. Press, Oxford, 1991).

3. Hamilton, W. D. The moulding of senescence by natural selection. J. Theor. Biol. 12, 12-45 (1966).

4. Stearns, S. C. The Evolution of Life Histories (Oxford Univ. Press, Oxford, 1992).

Partridge, L. \& Barton, N. H. Optimality, mutation and the evolution of ageing. Nature 362, 305-311 (1993).

6. Partridge, L. \& Barton, N. H. On measuring the rate of ageing. Proc. R. Soc. Lond. B 263, 1365-1371 (1996)

7. Charlesworth, B. Evolution in Age-structured Populations 2nd edn (Cambridge Univ. Press, Cambridge, 1994).

Finch, C. E. Longevity, Senescence and the Genome (Chicago Univ. Press, Chicago, 1990).

9. Bell, G. Evolutionary and nonevolutionary theories of senescence. Am. Nat. 124, 600-603 (1984).

10. Abrams, P. A. Does increased mortality favor the evolution of more rapid senescence? Evolution 47 , 877-887 (1993)

11. Sohal, R. S. in Insect Ageing (eds Collatz, K.-G. \& Sohal, R. S.) 23-44 (Springer, Berlin, 1986).

12. Hölldobler, B. \& Wilson, E. O. The Ants (Springer, Berlin, 1990).

13. Promislov, D. E. L. Senescence in natural populations of mammals: a comparative study. Evolution 45, 1869-1887 (1991)

14. Felsenstein, J. Phylogenies and the comparative method. Am. Nat. 125, 1-15 (1985).

15. Harvey, P. H. \& Pagel, M. D. The Comparative Method in Evolutionary Biology (Oxford Univ. Press, Oxford, 1991).

16. Purvis, A. \& Rambaut, A. Comparative analysis by independent constrast (CAIC): an Apple Macintosh application for analysing comparative data. Comp. Appl. Biosci. 11, 247-251 (1995).

17. Keller, L. (ed.). Queen Number and Sociality in Insects (Oxford Univ. Press, Oxford, 1993).

18. Keller, $\mathrm{L}$. The assessment of reproductive success of queens in ants and other social insects. Oikos 67, 177-180 (1993).

19. Keller, L. Social life: the paradox of multiple-queen colonies. Trends Ecol. Evol. 10, 355-360 (1995).

20. Bourke, A. F. G. \& Franks, N. R. Social Evolution in Ants (Princeton Univ. Press, Princeton, 1995).
21. Ross, K. G. \& Keller, L. Ecology and evolution of social organization-insights from fire ants and other highly eusocial insects. Ann. Rev. Ecol. Syst. 26, 631-656 (1995)

2. Nonacs, P. Queen number in colonies of social Hymenoptera as a kin-selected adaption. Evolution 42, $566-580$ (1988).

23. Reeve, H. K. \& Nonacs, P. Within-group aggression and the value of group members-theory and a field test with social wasps. Behav. Ecol. 8, 75-82 (1997).

24. Promislow, D. E. L. Senescence in natural populations of mammals: a comparative study. Evolution 45, 1869-1887 (1991).

25. Partridge, L. \& Fowler, K. Direct and correlated responses to selection on age at reproduction in Drosophila melanogaster. Evolution 46, 76-91 (1992).

26. Jarvis, J. U. M. \& Bennett, N. C. in The Biology of the Naked Mole-rat (eds Sherman, P. W., Jarvis, J. U. M. \& Alexander, R. D.) 66-69 (Princeton Univ. Press, Princeton, 1991)

27. Purvis, A., Gittleman, J. L. \& Luh, H.-K. Truth or consequences: effects of phylogenetic accuracy on two comparative methods. J. Theor. Biol. 167, 293-300 (1994).

28. Garland, T. J., Harvey, P. H. \& Ives, A. R. Procedures for the analysis of comparative data using independent contrasts. Syst. Biol. 41, 18-32 (1992).

29. Ridley, M. Mating frequency and fecundity in insects. Biol. Rev. 63, 509-549 (1988).

30. Ridley, M. Clutch size and mating frequency in parasitic Hymenoptera. Am. Nat. 142, 893-910 (1993).

Acknowledgements. We thank P. Abrams, A. Bourke, W. Brown, M. Chapuisat, B. Charlesworth, M. Milinski, L. Partridge, N. Perrin, F. Ratnieks, M. Rose, K. Reeve, M. Ridley and S. Stearns for comments on the manuscript, and S. Cover for constructing the ant phylogeny. This work was funded by grants from the Swiss NSF (L.K.).

Correspondence and requests for materials should be addressed to L.K. (e-mail: Laurent.keller@izea. unil.ch)

\section{The lateral line can mediate rheotaxis in fish}

\section{John C. Montgomery, Cindy F. Baker \& Alexander G. Carton}

Experimental Biology Research Group, School of Biological Sciences, University of Auckland, Private Bag 92019 Auckland, New Zealand

Rheotaxis is a behavioural orientation to water currents ${ }^{1}$. It has been demonstrated physiologically that some lateral-line receptors are particularly well suited to provide information on water currents ${ }^{2}$, but their contribution to rheotaxis has been largely overlooked. The accepted view is that rheotaxis is mediated by visual and tactile cues", and that in rheotactic orientation "the lateral lines play only a minor role"3. Here we provide a direct demonstration that rheotaxis can be mediated by the lateral line, and indeed by one specific receptor class of this system. In three diverse fish species, pharmacological block of the entire lateralline system substantially increases the velocity threshold for rheotactic behaviour. The same effect is observed when only superficial neuromasts are ablated, whereas blockade of the other receptor class, canal neuromasts, has no such effect. Our results therefore demonstrate that superficial neuromasts make an important contribution to rheotactic behaviour in fish.

Rheotaxis is an important and widespread behaviour in fish ${ }^{4}$. Examples include upstream migration, which counteracts the passive drift of eggs and larvae, and station-holding by facing upstream, which puts the fish in the best position to intercept food and odours carried in the current.

But what is the sensory basis of rheotaxis? A fish displaced by a current can use visual and tactile cues to initiate a response. Indeed, detection of the current has to be made against some stationary reference point. However, fish should also be able to detect current flow using the mechanosensory lateral-line system. This system, which is found in all fish, has receptors that are hair-cell patches called neuromasts. There are two main receptor classes within the lateral line: canal neuromasts, which are located within canals under the skin, and superficial neuromasts, which occur on the surface of the skin. Canal neuromasts respond best to water accelerations ${ }^{2}$, such as those produced by the movements of other animals, whereas electrophysiological recordings show that superficial neuromasts respond to the velocity of water movements over the skin of the $f_{i s h}{ }^{5}$. However, it has not been demonstrated clearly that the information from superficial neuromasts has an effect on the 